Pacific Journal of Mathematics

CHANGING SIGNS OF FOURIER COEFFICIENTS 


\title{
CHANGING SIGNS OF FOURIER COEFFICIENTS
}

\author{
R. E. EDWARDS
}

\begin{abstract}
Beginning with a mild extension of a theorem of Littlewood, as generalised by Helgason and by Grothendieck from the circle to a general compact Abelian group $G$, we derive some properties of the Fourier series of continuous functions on $G$ in relation to arbitrary changes of sign of the coefficients. The main result of this latter type sharpens a fact known for the circle by showing that a continuous function $f$ on $G$ and a \pm 1 -valued function $\omega$ on the character group $X$ may be chosen so that

$$
T_{\omega} f=\Sigma_{\xi \in X} \omega(\xi) \hat{f}(\xi) \xi
$$

belongs to no Orlicz space $L_{\Lambda}(G)$ for which $\lim _{u \rightarrow \infty} u^{-2} \Lambda(u)=\infty$. Similar results are obtained which apply when $f$ is assumed to be merely integrable: in this case one can assert little more than that $T_{\omega} f$ is a pseudomeasure on $G$.
\end{abstract}

Notation. With the sole exception of (3.5), $G$ denotes a compact Abelian group and $X$ its character group. We write $\Omega$ for the set of all functions on $X$ taking only the values \pm 1 , and $\Omega^{*}$ for the set of all complex-valued functions on $X$ having absolute value everywhere equal to 1 .

The symbol $L^{p}(G)$ denotes the usual Lebesgue space formed with the Haar measure on $G$, and likewise for $l^{p}(X)$ and the (purely discontinuous) Haar measure on $X . M(G)$ is the space of complex Radon measures on $G$, and $C(G)$ the space of complex-valued continuous functions on $G$ with the usual sup norm.

1. A Littlewood-type theorem.

(1.1) THEOREM. If $F$ is a complex-valued function on $X$ with the property that for each $\omega \in \Omega$ the series

$$
\Sigma \omega(\xi) F(\xi) \xi
$$

is a Fourier-Stieltjes series, then $F \in l^{2}(X)$.

(1.2) Remarks. If $G$ is the circle group, and if the Fourier series are taken in their "real" form, the stated conclusion is known to follow from the hypothesis that each series (1.1.1) is a Fourier series: see [7], p. 215, where a proof is based upon the properties of Rademacher series. One difficulty attending an extension of this

Received April 27, 1964. 
approach to general $G$, using now the properties of Rademacher series in relation to general orthogonal expansions (see [5], pp. 179-186), is the absence of detailed information regarding the pointwise a.e. summability of Fourier series on groups. For this reason we adopt an approach which side-steps this issue and which is due to Helgason and to Grothendieck independently.

Helgason [3] proves that the conclusion $F \in l^{2}(X)$ follows from the assumption that (1.1.1) is a Fourier series for each $\omega \in \Omega^{*}$. The same conclusion, but with $\Omega$ reinstated, is implicit in Grothendieck [1] (especially p. 90) and [2]. Our proof of (1.1) will begin from Helgason's version.

(1.3) Proof of (1.1). Notice that it is enough to consider realvalued functions $F$ in (1.1). For, if $F=U+i V$ ( $U, V$ real valued) is a Fourier-Stieltjes transform, so also is $\bar{F}=U-i V$, and therefore also each of $U$ and $V$. So, by virtue of Lemma (1.5) infra, if (1.1.1) is a Fourier-Stieltjes series for each $\omega \in \Omega$, then the same is true when $\omega$ is replaced by any bounded, complex-valued function on $X$, and hence in particular whenever $\omega \in \Omega^{*}$.

This being so, if $f \in L^{1}(G)$, the series

$$
\Sigma \omega(\xi) F(\xi) \hat{f}(\xi) \xi
$$

is a Fourier series for each $\omega \in \Omega^{*}$, and Helgason's theorem affirms then that $F \hat{f} \in l^{2}(X)$. Hence (by the closed graph theorem, for example)

$$
\|F \hat{f}\|_{2} \leqq \text { const. }\|f\|_{1}
$$

for each $f \in L^{1}(G)$. If $f$ is permitted to vary over an approximate identity in $L^{1}(G)$, (1.1.2) shows at once that $F \in l^{2}(X)$.

\section{(1.4) COROLlaRY. If the series}

$$
\Sigma c(\xi) F(\xi) \xi
$$

is a Fourier-Stieltjes series for each complex-valued function $c$ on $X$ which tends to zero at infinity, then $F \in l^{2}(X)$.

Proof. Theorem (1.1) implies at once that $c F \in l^{2}(X)$ for each $c$ of the type specified. But then, since these $c$ 's form a Banach space, the closed graph theorem shows that

$$
\|c F\|_{2} \leqq \text { const. } \sup _{\xi}|c(\xi)|,
$$

whence it follows directly that $F \in l^{2}(X)$.

It remains to prove the following lemma. In it, $R^{x}$ denotes the 
space of all real-valued functions on $X$ endowed with the product topology.

(1.5) Lemma. Let $E$ be a topological vector space which is the union of an increasing sequence $\left(A_{n}\right)_{n=1}^{\infty}$ of compact, convex and balanced subsets. Let $X$ be any set, and let $\Phi$ be any bounded subset of $R^{X}$ with the following properties-

(a) $\Phi$ is a nonmeagre subspace of $R^{x}$ (as is the case if $\Phi$ is locally compact or complete metrisable);

(b) $\Omega \cdot \Phi \subset \Phi$; and if $X_{0}$ is a finite subset of $X, \varphi_{i} \in \Phi(i=1,2)$, and $\varphi=\varphi_{1}$ on $X_{0}, \varphi=\varphi_{2}$ on $X \cap X_{0}^{\prime}$, then $\varphi \in \Phi$.

Suppose finally that $T$ is a continuous linear mapping of $E$ into $R^{x}$ such that

(c) $T(E)$ contains $\Phi$ and also all characteristic functions of onepoint subsets of $X$.

Then there exists a natural number $n$ and a number $r \geqq 0$ such that $r \cdot T\left(A_{n}\right)$ contains every $\psi \in R^{X}$ satisfying $|\psi| \leqq \varphi$ for some $\varphi \in \Phi$.

Proof. This is presented in two steps.

(1) The compactness of $A_{n}$ and the continuity of $T$ ensure that $T\left(A_{n}\right)$ is closed in $R^{x}$. By (c), $\Phi$ is the union of the sets $T\left(A_{n}\right) \cap \Phi$, each of which is closed in $\Phi$. By (a), therefore, there exists a natural number $n^{\prime}$ such that $T\left(A_{n^{\prime}}\right) \cap \Phi$ contains a nonvoid open subset of $\Phi$. That is, there exists $\varphi_{0} \in \Phi$ and a finite subset $X_{0}$ of $X$ such that $T\left(A_{n^{\prime}}\right)$ contains each $\varphi \in \Phi$ coinciding on $X_{0}$ with $\varphi_{0}$. Enumerate $X_{0}$ as $\left\{\xi_{j}: j=1, \cdots, k\right\}$ and let $\varphi_{j}$ be the characteristic function of $\left\{\xi_{j}\right\}_{\text {. }}$ By (c) once more, there exists $e_{j} \in E$ such that $T\left(e_{j}\right)=\varphi_{j}$. For any $\varphi \in \Phi$ we can write

$$
\varphi=\sum_{j=1}^{k}\left[\varphi\left(\xi_{j}\right)-\varphi_{0}\left(\xi_{j}\right)\right] \varphi_{j}+\varphi^{*},
$$

where, thanks to (b), $\varphi^{*} \in \Phi$. Then

$$
\varphi=T\left(\sum_{j=1}^{k}\left[\varphi\left(\xi_{j}\right)-\varphi_{0}\left(\xi_{j}\right)\right] e_{j}+e^{*}\right)
$$

for some $e^{*} \in A_{n^{\prime}}$. Since $\Phi$ is bounded, there exists a number $r^{\prime}$, independent of $\varphi$, such that

$$
\sum_{j=1}^{k}\left|\varphi\left(\xi_{j}\right)-\varphi_{0}\left(\xi_{j}\right)\right| \leqq r^{\prime}
$$

Moreover, there exists an $n^{\prime \prime}$ such that $A_{n^{\prime \prime}}$ contains all the $e_{j}$. It thus appears that, if we set $r=r^{\prime}+1$ and $n=\max \left(n^{\prime}, n^{\prime \prime}\right)$, then $\Phi \subset r \cdot T\left(A_{n}\right)$. 
(2) Take now any $\theta \in R^{x}$ having a finite support and any $\psi^{\prime} \in R^{x}$ satisfying $|\psi| \leqq \varphi_{0}$ for some $\varphi_{0} \in \Phi$. For a suitable choice of $\omega \in \Omega$ we have then (the sums extending over all $\xi \in X$ )

$$
\begin{aligned}
|\Sigma \theta(\xi) \psi(\xi)| & \leqq \Sigma|\theta(\xi) \psi(\xi) \leqq \Sigma| \theta(\xi) \mid \varphi_{0}(\xi) \\
& =\left|\Sigma \theta(\xi) \varphi_{0}(\xi) \omega(\xi)\right| \\
& \leqq \operatorname{Sup}_{\varphi \in \Phi}|\Sigma \theta(\xi) \varphi(\xi)| \\
& \leqq \operatorname{Sup}_{e \in \boldsymbol{A}_{n}}|\Sigma \theta(\xi) T e(\xi)|
\end{aligned}
$$

the last two steps by (b) and by (1) respectively. The bipolar theorem shows now that $\psi$ is adherent in $R^{x}$ to $T\left(r A_{n}\right)$. But this last set is closed in $R^{x}$, and so $\psi \in r \cdot T\left(A_{n}\right)$, as alleged.

(1.6) REMARK. To apply (1.5) in the situation prevailing in (1.3), we take $E=M(G)$ with the topology $\sigma(M(G), C(G)), A_{n}$ to be the closed ball in $M(G)$ of radius $n, \Phi=F \cdot \Omega$ (which is compact for the product topology), and $T \mu=\widehat{\mu}$ for $\mu \in E$. Then (c) is satisfied when (1.1.1) is a Fourier-Stieltjes series for each $\omega \in \Omega$, and the desired conclusion follows.

Notice that we might also take $E=L^{p}(G)$ with $1<p \leqq \infty$ and use the weak topology $\sigma\left(L^{p}, L^{p^{\prime}}\right)$. It would then follow that, if (1.1.1) is the Fourier series of a function in $L^{p}(G)$ whenever $\omega \in \Omega$, then the same is true when $\omega$ is replaced by any bounded real-valued function on $X$.

2. Fourier coefficients of continuous functions. We shall henceforth write $T(G)$ for the space of all trigonometric polynomials on $G$, and $T_{0}(G)$ for the set of such polynomials

$$
t(x)=\Sigma c_{k} \xi_{k}(x)
$$

(a finite sum)

with the property that, for some choice of the \pm signs (depending upon $t$ ),

$$
\max _{x \in G}\left|\Sigma \pm c_{k} \xi_{k}(x)\right| \leqq 1 .
$$

If for $\omega \in \Omega$ we define the functional operator $T_{\omega}$ for (say) $f \in C(G)$ by setting

$$
T_{\omega} f=\Sigma \omega(\xi) \hat{f}(\xi),
$$

the series converging in $L^{2}(G), T_{0}(G)$ consists precisely of those $t \in T(G)$ such that

$$
\min _{\omega \in \Omega}\left\|T_{\omega} t\right\| \leqq 1
$$


Expressed differently, $T_{0}(G)$ consists of all functions $T_{\omega} t$ obtained when $\omega$ varies over $\Omega$ and $t$ over all trigonometric polynomials satisfying $\|t\| \leqq 1$. The operators $T_{\omega}$ will appear again later in this section.

We begin with a result which characterises those functions $F$ on $X$ for which $F \hat{f} \in l^{1}(X)$ for each $f \in C(G)$.

(2.1) THEOREm. Let $F$ be a complex-valued function on $X$.

(a) If the series $\Sigma \omega(\xi) F(\xi) \hat{f}(\xi)$ is convergent whenever $\omega \in \Omega$ and $f \in C(G) \cdots$ i.e., if

$$
\Sigma|F(\xi) \hat{f}(\xi)|<\infty
$$

for each $f \in C(G) \cdots$ then $F \in l^{2}(X)$.

(b) There exists a number $M$ such that

$$
\|F\|_{2} \leqq M \cdot \operatorname{Sup}\left\{\|F \hat{f}\|_{1}: f \in C(G),\|f\| \leqq 1\right\} .
$$

(c) There exists a number $M$ such that for all $F \in l^{2}(X)$ one has

$$
\|F\|_{2} \leqq M \cdot \operatorname{Sup}\left\{|\Sigma F(\xi) \hat{t}(\xi)|: t \in T_{0}(G)\right\} .
$$

Proof. (a) In view of the Riesz representation theorem, the hypothesis of (a) entails that each series (1.1.1) is a Fourier-Stieltjes series, so that the assertion follows from Theorem (1.1).

(b) The proof of this is similar to, and simpler than, that of (c). It is left to the reader.

(c) On $l^{2}(X)$ define the norm

$$
\begin{aligned}
N(F) & =\operatorname{Sup}\left\{|\Sigma F(\xi) \hat{t}(\xi)|: t \in T_{0}(G)\right\} \\
& =\operatorname{Sup}\{|\Sigma \omega(\xi) F(\xi) \hat{t}(\xi)|: t \in T(G),\|t\| \leqq 1, \omega \in \Omega\} .
\end{aligned}
$$

It is quite evident that $N(F) \leqq\|F\|_{2}$. By virtue of the inversion theorem for Banach spaces, it will therefore suffice to show that $l^{2}(X)$ is complete for $N$.

To this end, assume that $\left(F_{n}\right)_{n=1}^{\infty}$ is an N-Cauchy sequence in $l^{2}(X)$. Evidently, $F=\lim F_{n}$ exists pointwise on $X$. Given $\varepsilon>0$, there exists $n_{0}=n_{0}(\varepsilon)$ such that

$$
\operatorname{Sup}_{t \omega}\left|\Sigma \omega(\xi)\left[F_{m}(\xi)-F_{n}(\xi)\right] \hat{t}(\xi)\right| \leqq \varepsilon
$$

whenever $m, n \geqq n_{0}$. Hence, since each $\hat{t}$ has a finite support,

$$
\operatorname{Sup}_{t, \omega}\left|\Sigma \omega(\xi)\left[F(\xi)-F_{n}(\xi)\right] \hat{t}(\xi)\right| \leqq \varepsilon
$$

for $n \geqq n_{0}$. In particular, if $n^{\prime}=n_{0}(1)$,

$$
\operatorname{Sup}_{t, \omega}|\Sigma \omega(\xi) F(\xi) \hat{t}(\xi)| \leqq N\left(F_{n^{\prime}}\right)+1 \text {. }
$$


Separating real and imaginary parts and then choosing $\omega \in \Omega$ suitably for each of these, it follows that

$$
\operatorname{Sup}_{t} \Sigma|F(\xi) \hat{t}(\xi)| \leqq 2 N\left(F_{n^{\prime}}\right)+2,
$$

the supremum now being taken over the unit ball of $T(G)$. This last is dense in the unit ball of $C(G)$, and so we may infer that $F \hat{f} \in l^{1}(X)$ for each $f \in C(G)$. By (a), therefore, $F \in l^{2}(X)$. Knowing this, (2.1.4) signifies exactly that $N\left(F-F_{n}\right) \leqq \varepsilon$ for $n \geqq n_{0}$. Completeness is thus established.

(2.2) REMARKs. (i) It is scarcely necessary to point out that the conditions on $F$ in order that the series $\Sigma F(\xi) \hat{f}(\xi)$ be convergent for each $f \in C(G)$ are of quite a different sort. For example, if $G$ is the circle group, and if $\left(\alpha_{n}\right)_{n=0}^{\infty}$ is a sequence which is convex and such that $\alpha_{n} \log n \rightarrow 0$ as $n \rightarrow \infty$, then the series

$$
\sum_{n=-\infty}^{\infty} \alpha_{|n|} e^{i n x}
$$

is convergent in $L^{1}(G)$ ([7], pp. 183-185). Consequently the series

$$
\sum_{n=-\infty}^{\infty} \alpha_{|n|} \hat{f}(n) e^{i n x}
$$

is convergent for each $x \in G$ if $f \in L^{\infty}(G)$, and uniformly with respect to $x$ if $f \in C(G)$. Yet, of course,

$$
\sum_{n=-\infty}^{\infty}\left|\alpha_{|n|}\right|^{2}
$$

is generally divergent.

(ii) I am grateful to a referee for pointing out that Theorem 2.1 (a) was proved for the circle by Sidon [6] and for all compact groups (Abelian or not) by Helgason [4].

The following is a simple extension of (2.1.b), the proof of which is left to the reader.

(2.3) CoRollaRy. If $1 \leqq p \leqq 2$, and if $F \hat{f} \in l^{p}(X)$ for each $f \in C(G)$, then $F \in l^{q}(X)$ where $q=2 p /(2-p)$. Moreover, there exists a number $M$ such that for all complex-valued functions $F$ on $X$ one has

$$
\|F\|_{q} \leqq M \cdot \operatorname{Sup}\left\{\|F \hat{f}\|_{p}: f \in C(G),\|f\| \leqq 1\right\} .
$$

The next result deals with the possibility of expanding arbitrary functions in $L^{2}(G)$ as a series of scalar multiples of elements of $T_{0}(G)$. 
(2.4) THEOREM. There exists a number c such that each $h \in L^{2}(G)$ is expressible in the form

$$
h=\sum_{n=1}^{\infty} \lambda_{n} t_{n}^{0},
$$

where $t_{n}^{0} \in T_{0}(G)$, or alternatively in the form

$$
h=\sum_{n=1}^{\infty} \lambda_{n} T_{\omega_{n}} t_{n},
$$

where $\omega_{n} \in \Omega$ and $t_{n} \in T(G),\left\|t_{n}\right\| \leqq 1$; and where in either case

$$
\sum_{n=1}^{\infty}\left|\lambda_{n}\right| \leqq c \cdot\|h\|_{2} \cdot
$$

Proof. By (2.1.3) and Parseval's formula we have for $g \in L^{2}(G)$ the inequality

$$
\|g\|_{2} \leqq M \cdot \operatorname{Sup}\left\{\left|\int_{G} g t d x\right|: t \in T_{0}(G)\right\} .
$$

According to the bipolar theorem, it follows that any $h \in L^{2}(G)$ satisfying $\|h\|_{2} \leqq M^{-1}=r$ belongs to the closed, convex, balanced envelope in $L^{2}(G)$ of $T_{0}(G)$. The rest is a direct application of the general but simple result contained in the following lemma.

(2.5) Lemma. Let $E$ be a normed vector space, $A$ a subset of $E$, and $B$ the convex, balanced envelope in $E$ of $A$. Suppose that there exists a number $r>0$ such that $B$ is dense in the ball $\|x\| \leqq r$ in $E$. Let $\varepsilon>0$. Then any $x \in E$ satisfying $\|x\| \leqq r$ can be represented as a sum

$$
x=\sum_{n=1}^{\infty} \lambda_{n} a_{n},
$$

where $a_{n} \in A(n=1,2, \cdots)$ and where the scalars $\lambda_{n}$ satisfy the condition

$$
\sum_{n=1}^{\infty}\left|\lambda_{n}\right| \leqq 1+\varepsilon
$$

Proof. Choose numbers $\varepsilon_{n}>0$ such that $\varepsilon_{1}=1$ and

$$
\sum_{n=1}^{\infty} \varepsilon_{n} \leqq 1+\varepsilon .
$$

Then elements $b_{n}$ of $B$ may be chosen so that

$$
\left\|x-\sum_{i=1}^{n} \varepsilon_{i} b_{i}\right\| \leqq r \varepsilon_{n+1} \text {. }
$$


Indeed, we choose $b_{1} \in B$ so that $\left\|x-b_{1}\right\|=\left\|x-\varepsilon_{1} b_{1}\right\| \leqq r \varepsilon_{2}$; this is possible by the density assertion concerning $B$. If $b_{1}, \cdots, b_{n}$ have been chosen in accord with (2.5.1), we have

$$
\left\|\varepsilon_{n+1}^{-1}\left(x-\sum_{i=1}^{-1} \varepsilon_{i} b_{i}\right)\right\| \leqq r
$$

and so, by the same hypothesis concerning $B$, one may select $b_{n+1} \in B$ so that

$$
\left\|\varepsilon_{n+1}^{-1}\left(x-\sum_{i=1}^{n} \varepsilon_{i} b_{i}\right)-b_{n+2}\right\| \leqq r \varepsilon_{n+1}^{-1} \varepsilon_{n+2} .
$$

On rearrangement this gives (2.5.1) with $n$ replaced by $n+1$, and the construction of the sequence $\left(b_{n}\right)_{n=1}^{\infty}$ proceeds by recurrence.

It now suffices to write

$$
b_{n}=\sum_{k=1}^{\infty} \lambda_{n k} a_{n k}
$$

where the $a_{n k} \in A, \lambda_{n k}=0$ for $k>k_{n}$, and $\sum_{k=1}^{\infty}\left|\lambda_{n k}\right| \leqq 1$. The desired representation of $x$ follows on arranging the $a_{n k}$ and the $\lambda_{n k}$ as single sequences $\left(a_{n}\right)_{n=1}^{\infty}$ and $\left(\lambda_{n}\right)_{n=1}^{\infty}$ for which

$$
\begin{aligned}
\sum_{n=1}^{\infty}\left|\lambda_{n}\right| & =\sum_{n=1}^{\infty} \sum_{k=1}^{\infty} \varepsilon_{n}\left|\lambda_{n k}\right| \\
& \leqq \sum_{n=1}^{\infty} \varepsilon_{n} \leqq 1+\varepsilon .
\end{aligned}
$$

Although the conclusion of Theorem (2.4) itself implies considerable irregularity in the behaviour of the $T_{\omega} t$ involved, this is best crystallised by reverting to Theorem (1.1), which leads to the following result.

(2.6) Theorem. Assume that $G$ is infinite, let $g \in L^{1}(G), g \notin L^{2}(G)$. Then there exist an $\omega \in \Omega$ and a sequence $\left(t_{n}\right)_{n=1}^{\infty}$ of trigonometric polynomials on $G$ such that

$$
\begin{gathered}
\left\|t_{n}\right\| \leqq 1, \\
\lim _{n \rightarrow \infty}\left|\int_{a}\left(T_{\omega} t_{n}\right) g d x\right|=\infty .
\end{gathered}
$$

Proof. The function $\hat{g}$ does not belong to $l^{2}(X)$ and so, by Theorem (1.1), there exists an $\omega \in \Omega$ such that $\Sigma \omega(\xi) \hat{g}(\xi) \xi$ is not a FourierStieltjes series. Taking any sequence $\left(r_{n}\right)_{n=1}^{\infty}$ of trigonometric polynomials such that $\left\|r_{n}\right\|_{1} \leqq 1$ and $\lim _{n \rightarrow \infty} \widehat{r}_{n}(-\xi)=1$ at all points of the countable support of $\hat{g}$, it follows that 


$$
\lim _{n}\left\|\Sigma \omega(\xi) \hat{g}(\xi) \hat{r}_{n}(-\xi) \xi\right\|_{1}=\infty .
$$

Hence there exists an $f \in C(G)$ such that

$$
\lim \sup _{n}\left|\Sigma \omega(\xi) \hat{g}(\xi) \hat{r}_{n}(-\xi) \hat{f}(-\xi)\right|=\infty .
$$

By dropping terms, we may replace the lim sup by lim, and then it suffices to take $t_{n}=\left(r_{n} * f\right) /\|f\|$.

(2.7) Remarks. From (2.6) it follows that the sequence $\left(t_{n}\right)_{n=1}^{\infty}$ may be chosen so as to satisfy (2.6.1) and

$$
\lim _{n}\left\|T_{\omega} t_{n}\right\|_{p}=\infty(\forall p>2) ;
$$

or so as to satisfy (2.6.1) and to be such that the $T_{\omega} t_{n}$ are unbounded in any given Orlicz space $L_{\Lambda}(G)$ defined by a function $\Lambda$ such that $\lim _{u \rightarrow \infty} u^{-2} \Lambda(u)=\infty$ (see [7], pp. 170-175).

From (2.6) we may derive in turn the anomalous behaviour of the $T_{\omega} f$ with $f$ continuous.

(2.8) THEOREM. Assuming again that $G$ is infinite, take any $g \in L^{1}(G), g \notin L^{2}(G)$. Then there exist an $\omega \in \Omega$ and an $f \in C(G)$ such that

$$
\int_{G}\left|\left(T_{\omega} f\right) \cdot g\right| d x=\infty
$$

Proof. Choose $\omega$ as in (2.6). If the conclusion of the present theorem were false, one would have a linear map $f \rightarrow\left(T_{\omega} f\right) \cdot g$ of $C(G)$ into $L^{1}(G)$. An easy application of the closed graph theorem would show that this map is continuous. But this would contradict (2.6).

(2.9) REmarks. (a) The preceding proof could be refined so as to show that the set of $\omega \in \Omega$, for which

$$
\mathscr{F}_{\omega}=\left\{f \in C(G):\left(T_{\omega} f\right) \cdot g \in L^{1}(G)\right\}
$$

is second category in $C(G)$, is first category in $\Omega$; or (to put it another way) that for a residual (=complement of a first category) set of $\omega \in \Omega, \mathscr{F}_{\omega}$ is first category in $C(G)$.

(b) From (2.8) it follows that $\omega \in \Omega$ and $f \in C(G)$ can be found so that $T_{\omega} f$ fails to belong to $\bigcup\left\{L^{p}(G): p>2\right\}$, or indeed to any Orlicz space $L_{\Lambda}(G)$ for which $\lim _{u \rightarrow \infty} u^{-2} \Lambda(u)=\infty$ (see (2.7)).

With this in mind one may compare (2.8) with a known analogous result for the circle group and Fourier series handled in their "real" form. From the results on pp. 214-215 of [7] it follows that for any fixed $p>2$ there exist a function $f$ such that 


$$
\int_{\theta} \exp \left(\alpha|f|^{2}\right) d x<\infty(\forall \alpha)
$$

and a sequence of \pm 1 's such that, writing

$$
f \sim 1 / 2 a_{0}+\sum_{n=1}\left(a_{n} \cos n x+b_{n} \sin n x\right),
$$

the series

$$
\pm 1 / 2 a_{0}+\sum_{n=1}^{\infty} \pm\left(a_{n} \cos n x+b_{n} \sin n x\right)
$$

is not the Fourier series of any function in $L^{p}(G)$.

Evidently, Theorem (2.8) forms an analogue of this which has been sharpened in two directions: first by the enlargement of $L^{p}(G)$ to the space of functions whose product with $g$ is integrable, and second by the narrowing of the class defined by (2.9.1) [which contains essentially unbounded functions] to $C(G)$.

3. Related results. Helgason [3] and Grothendieck ([1], p. 90) obtained (again independently) the following result.

(3.1) THEOREM. If a function $F$ on $X$ has the property that (1.1.1) is the Fourier series of a function in $L^{\infty}(G)$ whenever $\omega \in \Omega$, then $F \in l^{1}(X)$.

On the basis of (1.6) this result is easily established. For suppose more generally that (1.1.1) is the Fourier series of a function in $L^{p}(G)$ whenever $\omega \in \Omega$, where $1<p \leqq \infty$. Then, by (1.6),

$$
\Sigma u(\xi) F(\xi) \xi
$$

is the Fourier series of a function in $L^{p}(G)$ whenever $u \in l^{\infty}(X)$. Taking any $f \in L^{p^{\prime}}(G)$, one obtains therefore a linear map $T: u \rightarrow \Sigma u(\xi) F(\xi) \hat{f}(\xi) \xi$ of $c_{0}(X)$ into $C(G)$, and the closed graph theorem shows that $T$ is continuous. The adjoint $T^{\prime}$ maps $M(G)$ into $l^{1}(X)$. A simple calculation shows that $T^{\prime}$ carries $\varepsilon$ (the Dirac measure at the neutral element of $G)$ into $F \hat{f}$, so that $F \hat{f} \in l^{1}(X)$. A second application of the closed graph theorem shows that

$$
\Sigma|F(\xi) \hat{f}(\xi)| \leqq \text { const. }\|f\|_{p^{\prime}} \text {. }
$$

Conversely, if (3.1.1) holds, it is easy to verify that (1.1.1) is the Fourier series of a function in $L^{p}(G)$ whenever $\omega \in \Omega$. Now, if $p=\infty$, then $p^{\prime}=1$ and (3.1.1) entails that $F \in l^{1}(X)$. This proves (3.1).

(3.2) CoRollary. Suppose given any $g \in L^{1}(G)$ such that $\hat{g} \notin l^{1}(X)$. 
Then there exists an $\omega \in \Omega$ with the following property: for any net $\left(r_{i}\right)$ of functions in $L^{1}(G)$ such that

(i) $\hat{r}_{i} \in l^{1}(X)$, and

(ii) $\lim _{i} \hat{r}(\xi)=1$ for each $\xi \in X$ for which $\hat{g}(-\xi)=1$, one has

$$
\operatorname{Sup}_{i}\left|\int_{\theta} T_{\omega}\left(r_{i} * f\right) \cdot g d x\right|=\infty
$$

for each $f$ in a comeagre (and therefore dense) subset of $L^{1}(G)$.

If it be assumed that $g \in L^{2}(G)$, one may in place of (i) assume merely that each $r_{i} \in L^{2}(G)$.

Proof. According to Theorem (3.1), there exists an $\omega \in \Omega$ such that $\Sigma \omega(\xi) \hat{g}(-\xi) \xi$ is not the Fourier series of a function in $L^{\infty}(G)$. Consequently we must have

$$
\operatorname{Sup}_{i}\left\|\Sigma \omega(\xi) \hat{g}(-\xi) \hat{r}_{i}(\xi) \xi\right\|_{\infty}=\infty \text {. }
$$

Consequently, too, the set of $f \in L^{1}(G)$, for which

$$
\operatorname{Sup}_{i}\left|\Sigma \omega(\xi) \hat{g}(-\xi) \hat{r}_{i}(\xi) \hat{f}(\xi)\right|<\infty
$$

must be meagre (first category) in $L^{1}(G)$. By Baire's theorem, any comeagre subset of $L^{1}(G)$ is dense therein. Since the left hand sides of (3.2.1) and (3.2.2) are plainly equal, the proof is complete.

(3.3) Remarks. (i) Since the support of $\hat{g}(-\xi)$ is an any case countable, we can for any given $f \in L^{1}(G)$ in the said comeagre set choose a sequence $\left(r_{n}\right)_{n=1}^{\infty}$ satisfying (3.2.i) and for which

$$
\lim _{n}\left|\int_{G} T_{\omega}\left(r_{n} * f\right) \cdot g d x\right|=\infty .
$$

(ii) From (i) it appears that one can choose always a sequence $\left(t_{n}\right)_{n=1}^{\infty}$ of trigonometric polynomials on $G$ such that $\left\|t_{n}\right\|_{1} \leqq 1$ and

$$
\lim _{n}\left|\int_{G} T_{\omega} t_{n} \cdot g d x\right|=\infty .
$$

(iii) Let $P(G)$ denote the space of pseudomeasures on $G$ (the dual of the space $A(G)$ of continuous functions $u$ on $G$ having absolutely convergent Fourier series, normed by $\left.\|u\|_{A}=\Sigma|\widehat{u}(\xi)|\right)$. Whilst it is evident that each $T_{\omega}$ defines a continuous linear map of $P(G)$ into itself, Corollary (3.2) says (roughly) that, for general $f \in L^{1}(G) \subset P(G)$ and $\omega \in \Omega, T_{\omega} f$ cannot be better behaved than a pseudomeasure.

More precisely, suppose $E$ is a vector space such that $T(G) \subset E \subset$ 
$L^{1}(G)$. Suppose further that $T_{\omega} f$ is definable as an element of $E^{*}$ (the algebraic dual of $E$ ) whenever $\omega \in \Omega$ and $f \in L^{1}(G)$, in such a way that

(a) $\left\langle t, T_{\omega} f\right\rangle=\Sigma \omega(\xi) \hat{f}(\xi) \hat{t}(-\xi)$ for $t \in T(G), \omega \in \Omega$, and $f \in L^{1}(G)$;

(b) for any fixed $\omega \in \Omega$, the family $\left(T_{\omega} f_{i}\right)$ is $\sigma\left(E^{*}, E\right)$-bounded whenever the family $\left(f_{i}\right)$ is bounded in $L^{1}(G)$.

Then necessarily $E \subset A(G)$. This conclusion follows from Corollary (3.2), if we choose for $\left(r_{i}\right)$ a net of trigonometric polynomials on $G$ forming an approximate identity in $L^{1}(G)$.

(3.4) Introduce now the pseudomeasures on $X$. Then (3.1) can be rephrased as follows: If $F$ is a function on $X$ such that $\omega F$ is a pseudomeasure on $X$ for each $\omega \in \Omega$, then $F \in l^{1}(X)$. Bearing in mind (1.6) once more, the next theorem may be regarded as an analogue of (3.1) applying to groups $G$ which are not necessarily compact.

(3.5) THeorem. Let $G$ be a locally compact Abelian group (not necessarily compact), $X$ its character group, and $\mu$ a Radon measure on $X$. If $f \mu$ is a pseudomeasure on $X$ for each $f \in C_{0}(X)$, then $\mu$ is a bounded Radon measure on $X$. (The converse is true and trivial.)

Proof. Let $N(f)$ denote the pseudomeasure norm of $f \mu$, so that

$$
N(f)=\operatorname{Sup}\left\{\left|\int_{X} \hat{g} f d \mu\right|: g \in L^{1}(G),\|g\|_{1} \leqq 1, \hat{g} \in C_{c}(X)\right\} ;
$$

$C_{c}(X)$ denotes the space of continuous functions on $X$ having compact supports. For a fixed $g$ of the type specified, $f \rightarrow\left|\int_{X} \hat{g} f d \mu\right|$ is evidently a continuous seminorm on $C_{0}(X)$, so that $N$ is lower semicontinuous and therefore continuous. Thus there exists a number $m$ such that

$$
\left|\int_{X} \hat{g} f d \mu\right| \leqq m\|g\|_{1} \cdot\|f\|
$$

for all $f \in C_{0}(X)$ and all $g \in L^{1}(G)$ such that $\hat{g}$ has a compact support. Taking the supremum with respect to $f$ satisfying $\|f\| \leqq 1$, it appears that

$$
\int_{X}|\hat{g}| d|\mu| \leqq m\|g\|_{1}
$$

for each $g$ of the type specified. At this stage we allow $g$ to vary along a suitably chosen approximate identity in $L^{1}(G)$ to conclude that

$$
\int_{x} d|\mu| \leqq m<\infty,
$$

showing that $\mu$ is a bounded measure. 


\section{REFERENCES}

1. A. Grothendieck, Résumé des résultats essentiels dans la théorie des produits tensoriels topologiques et des espaces nucléaires, Ann. Inst. Fourier IV (1952), 73-112.

2. ——, Résultats nouveaux dans la théorie des opérateurs linéaires, I, II, Comptes Rendus Acad. Sci. Paris 239 (1954), 577-579, 607-609.

3. S. Helgason, Multipliers of Banach algebras, Ann. of Math. (2) 64 (1956), 240-254.

4. - Lacunary Fourier series on noncommutative groups, Proc. Amer. Math. Soc. 9 (1958), 782-790.

5. S. Kaczmarz, and H. Steinhaus, Theorie der Orthogonalreihen. Chelsea Publishing Co. (1951).

6. S. Sidon, Ein Satz über die Fourierschen Reihen stetiger Funktionen, Math. Zeitschr. 34 (1932), 485-486.

7. A. Zygmund, Trigonometric series, I. Cambridge University Press (1959).

Institute of AdVANCED STUdies,

Australian National University 



\title{
PACIFIC JOURNAL OF MATHEMATICS
}

\author{
EDITORS
}

\author{
H. Samelson \\ Stanford University \\ Stanford, California \\ R. M. Blumenthal \\ University of Washington \\ Seattle, Washington 98105
}

\author{
J. DugundjI \\ University of Southern California \\ Los Angeles, California 90007 \\ Richard Arens \\ University of California \\ Los Angeles, California 90024
}

\section{ASSOCIATE EDITORS}
E. F. BECKENBACH
B. H. NEUMaNN
F. WOLF
K. YosIDA

\section{SUPPORTING INSTITUTIONS}

\author{
UNIVERSITY OF BRITISH COLUMBIA \\ CALIFORNIA INSTITUTE OF TECHNOLOGY \\ UNIVERSITY OF CALIFORNIA \\ MONTANA STATE UNIVERSITY \\ UNIVERSITY OF NEVADA \\ NEW MEXICO STATE UNIVERSITY \\ OREGON STATE UNIVERSITY \\ UNIVERSITY OF OREGON \\ OSAKA UNIVERSITY \\ UNIVERSITY OF SOUTHERN CALIFORNIA
}

\author{
STANFORD UNIVERSITY \\ UNIVERSITY OF TOKYO \\ UNIVERSITY OF UTAH \\ WASHINGTON STATE UNIVERSITY \\ UNIVERSITY OF WASHINGTON \\ AMERICAN MATHEMATICAL SOCIETY \\ CALIFORNIA RESEARCH CORPORATION \\ SPACE TECHNOLOGY LABORATORIES \\ NAVAL ORDNANCE TEST STATION
}

Mathematical papers intended for publication in the Pacific Journal of Mathematics should by typewritten (double spaced). The first paragraph or two must be capable of being used separately as a synopsis of the entire paper. It should not contain references to the bibliography. Manuscripts may be sent to any one of the four editors. All other communications to the editors should be addressed to the managing editor, Richard Arens, at the University of California, Los Angeles, California 90024.

50 reprints per author of each article are furnished free of charge; additional copies may be obtained at cost in multiples of 50 .

The Pacific Journal of Mathematics is published quarterly, in March, June, September, and December. Effective with Volume 13 the price per volume (4 numbers) is $\$ 18.00$; single issues, $\$ 5.00$. Special price for current issues to individual faculty members of supporting institutions and to individual members of the American Mathematical Society: $\$ 8.00$ per volume; single issues $\$ 2.50$. Back numbers are available.

Subscriptions, orders for back numbers, and changes of address should be sent to Pacific Journal of Mathematics, 103 Highland Boulevard, Berkeley 8, California.

Printed at Kokusai Bunken Insatsusha (International Academic Printing Co., Ltd.), No. 6, 2-chome, Fujimi-cho, Chiyoda-ku, Tokyo, Japan.

PUBLISHED BY PACIFIC JOURNAL OF MATHEMATICS, A NON-PROFIT CORPORATION

The Supporting Institutions listed above contribute to the cost of publication of this Journal, but they are not owners or publishers and have no responsibility for its content or policies. 


\section{Pacific Journal of Mathematics}

\section{Vol. 15, No. $2 \quad$ October, 1965}

Patrick Robert Ahern, On the generalized F. and M. Riesz theorem......... 373

A. A. Albert, On exceptional Jordan division algebras ................ 377

J. A. Anderson and G. H. Fullerton, On a class of Cauchy exponential

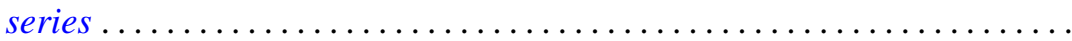

Allan Clark, Hopf algebras over Dedekind domains and torsion in

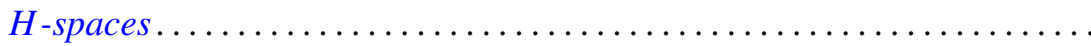

John Dauns and D. V. Widder, Convolution transforms whose inversion functions have complex roots .............................

Ronald George Douglas, Contractive projections on an $\mathrm{L}_{1}$ space ..........

Robert E. Edwards, Changing signs of Fourier coefficients ...............

Ramesh Anand Gangolli, Sample functions of certain differential processes on symmetric spaces .....................................

Robert William Gilmer, Jr., Some containment relations between classes of

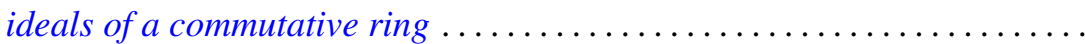

Basil Gordon, A generalization of the coset decomposition of a finite

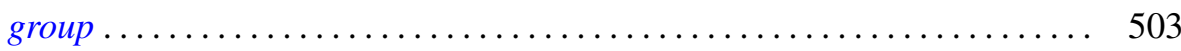

Teruo Ikebe, On the phase-shift formula for the scattering operator....... 511

Makoto Ishida, On algebraic homogeneous spaces ................ 525

Donald William Kahn, Maps which induce the zero map on homotopy ........ 537

Frank James Kosier, Certain algebras of degree one ................. 541

Betty Kvarda, An inequality for the number of elements in a sum of two sets of lattice points.................................

Jonah Mann and Donald J. Newman, The generalized Gibbs phenomenon for regular Hausdorff means. .

Charles Alan McCarthy, The nilpotent part of a spectral operator. II . ...

Donald Steven Passman, Isomorphic groups and group rings ...

R. N. Pederson, Laplace's method for two parameters .....

Tom Stephen Pitcher, A more general property than domination for sets of probability measures .............................

Arthur Argyle Sagle, Remarks on simple extended Lie algebras. .

Arthur Argyle Sagle, On simple extended Lie algebras over fields of

characteristic zero.

Tôru Saitô, Proper ordered inverse semigroups ...........

Oved Shisha, Monotone approximation

Indranand Sinha, Reduction of sets of matrices to a triangular form

Raymond Earl Smithson, Some general properties of multi-valued

functions .................................

John Stuelpnagel, Euclidean fiberings of solvmanifolds .... 